\title{
Riemann zeros and random matrix theory
}

\author{
N. C. Snaith \\ School of Mathematics, \\ University of Bristol, \\ Bristol BS8 1TW, UK
}

December 8, 2009

\begin{abstract}
In the past dozen years random matrix theory has become a useful tool for conjecturing answers to old and important questions in number theory. It was through the Riemann zeta function that the connection with random matrix theory was first made in the 1970s, and although there has also been much recent work concerning other varieties of $L$-functions, this article will concentrate on the zeta function as the simplest example illustrating the role of random matrix theory.
\end{abstract}

\section{Introduction}

The Riemann zeta function (see [35, 67]) is defined as a Dirichlet series or an Euler product over the primes,

$$
\zeta(s)=\sum_{n=1}^{\infty} \frac{1}{n^{s}}=\prod_{p}\left(1-1 / p^{s}\right)^{-1},
$$

which both converge for $\operatorname{Re}(s)>1$. These can be continued analytically, yielding a meromorphic function on the complex plane. There then exists a functional equation,

$$
\zeta(s)=\pi^{s-\frac{1}{2}} \frac{\Gamma\left(\frac{1}{2}-\frac{1}{2} s\right)}{\Gamma\left(\frac{1}{2} s\right)} \zeta(1-s),
$$

which relates the Riemann zeta function on one side of the critical line $\operatorname{Re}(s)=1 / 2$ to the same function on the other side. The connection with random matrix theory is through the infinite number of complex zeros that lie in the critical strip; that is, that have a real part between 0 and 1. The Riemann Hypothesis asserts that all these zeros have the form $\rho=1 / 2+i \gamma$ (with $\gamma$ real) - that they lie on the critical line with a real part equal to $1 / 2$. It is in the distribution of the imaginary parts of these zeros that we see the link with random matrix theory.

\section{Limiting zero statistics}

To begin, we will consider statistics of the zeros in the limit of large height on the critical line. If we label the Riemann zeros $\rho_{j}=\beta_{j}+i \gamma_{j}$, for those zeros with $\gamma_{j}>0$, then the 
density of the $\gamma_{j}$ 's increases with height $t$ up the critical line as

$$
d(t) \sim \frac{1}{2 \pi} \log \frac{t}{2 \pi} .
$$

It is the fluctuation of the positions of the zeros around this average density that is of interest, and so often it is useful to scale away the effect of the increasing density. We define a new set of points

$$
w_{j}=\gamma_{j} \frac{1}{2 \pi} \log \frac{\gamma_{j}}{2 \pi},
$$

which on average have a consecutive separation distance of one.

In the early 1970's Hugh Montgomery [57] studied two-point statistics of the Riemann zeros and conjectured that, for an appropriate test function $f(x)$,

\section{Conjecture 2.1 (Montgomery, 1973)}

$$
\lim _{W \rightarrow \infty} \frac{1}{W} \sum_{\substack{1 \leq n, m \leq W \\ n \neq m}} f\left(w_{n}-w_{m}\right)=\int_{-\infty}^{\infty} f(x) R_{2}(x) d x
$$

where

$$
R_{2}(x)=1-\left(\frac{\sin (\pi x)}{\pi x}\right)^{2} .
$$

He also proved that (2.3) holds true for $f(x)$ such that

$$
\hat{f}(\tau)=\int_{-\infty}^{\infty} f(x) e^{2 \pi i x \tau} d x
$$

has support in $(-1,1)$. Numerical evidence suggests that Montgomery's conjecture is correct [58], see Figure 1.

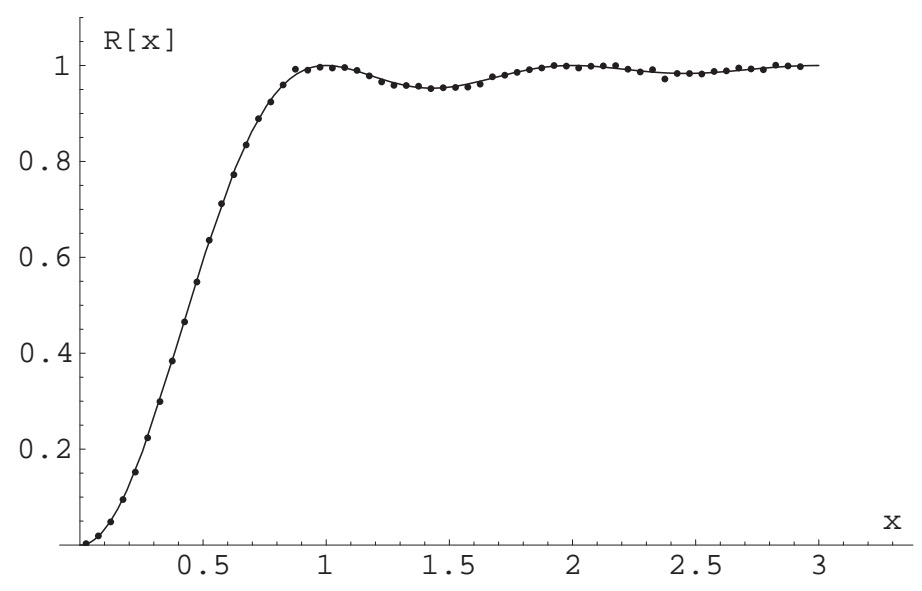

Figure 1: Two-point correlation function of the scaled Riemann zeros plotted using $10^{6}$ zeros around the $10^{20}$ th zero, computed by A. Odlyzko, and compared with $R_{2}(x)$ from $(2.4)$.

Shortly after Montgomery completed this work he was introduced to Freeman Dyson. After hearing about Montgomery's latest results Dyson [34] recognized in $R_{2}(x)$ the twopoint correlation function of eigenvalues of random unitary matrices defined, for a suitable test function $f(x, y)$, as: 


\section{Theorem 2.2}

$$
\begin{aligned}
\lim _{N \rightarrow \infty} \frac{1}{N} \int_{U(N)} & \sum_{\substack{1 \leq j, k \leq N \\
j \neq k}} f\left(\frac{N}{2 \pi}\left(\theta_{j}-\theta_{k}\right)\right) d A_{\text {Haar }} \\
& =\int_{-\infty}^{\infty} f(x)\left(1-\left(\frac{\sin \pi x}{\pi x}\right)^{2}\right) d x
\end{aligned}
$$

The integral in the first line is over the group $U(N)$ of unitary $N \times N$ matrices and $g(x)=$ $f\left(\frac{N}{2 \pi} x\right)$ is a $2 \pi$-periodic function. The matrices are weighted with respect to Haar measure on the group. $e^{i \theta_{1}}, e^{i \theta_{2}}, \ldots, e^{i \theta_{N}}$ are the eigenvalues of $A \in U(N)$.

As there are $N$ eigenangles $\left(\theta_{1}, \ldots, \theta_{N}\right)$ on a circle of circumference $2 \pi$, we can scale the eigenangles by $N / 2 \pi$ in order to give them an average consecutive spacing of unity. In (2.6) the test function $f(x)$ is being sampled at the spacings between pairs of scaled eigenangles and the result is averaged over the unitary group $U(N)$. This is equal (see, for example, [17] or [54]) to the second line of (2.6), which features the same two-point correlation function $R_{2}(x)$ as Montgomery's conjecture.

There is substantial further evidence, both analytic and numerical, that in the limit of large height up the critical line, statistics describing the distribution of the Riemann zeros converge, when correctly scaled, to the limiting distribution of eigenvalues of large unitary matrices $[6,8,43,58,62]$.

\section{Modelling zeta at finite height on the critical line}

Thus it appears that random matrix theory succeeds in modelling the limiting distribution of Riemann zeros, but this is not the end of the story. There is a theorem of Selberg (see eg. $[67,58])$

Theorem 3.1 (Selberg)

For any rectangle $B \in \mathbb{C}$,

$$
\begin{aligned}
\lim _{T \rightarrow \infty} \frac{1}{T} \mid\{t: T \leq t & \left.\leq 2 T, \frac{\log \zeta(1 / 2+i t)}{\sqrt{\frac{1}{2} \log \log T}} \in B\right\} \mid \\
& =\frac{1}{2 \pi} \iint_{B} e^{-\left(x^{2}+y^{2}\right) / 2} d x d y
\end{aligned}
$$

That is, in the limit as $T$, the height up the critical line, tends to infinity, the value distributions of the real and imaginary parts of $\log \zeta(1 / 2+i T) / \sqrt{(1 / 2) \log \log T}$ each tend, independently, to a Gaussian with unit variance and zero mean. Odlyzko [58] computed numerically the distribution of $\operatorname{Re} \log \zeta(1 / 2+i T) / \sqrt{(1 / 2) \log \log T}$ for values of the Riemann zeta function around the $10^{20}$ th zero and he obtained the solid curve in Figure 2. Despite the apparently large height on the critical line at which he did the computation, the value distribution is still far from the limiting Gaussian, shown in the short-dashed line on the figure.

To model this distribution using random matrix theory, some analogue of the Riemann zeta function is needed. We know that the zeros of the Riemann zeta function, at least in the limit of large height up the critical line, behave like the eigenvalues of large 


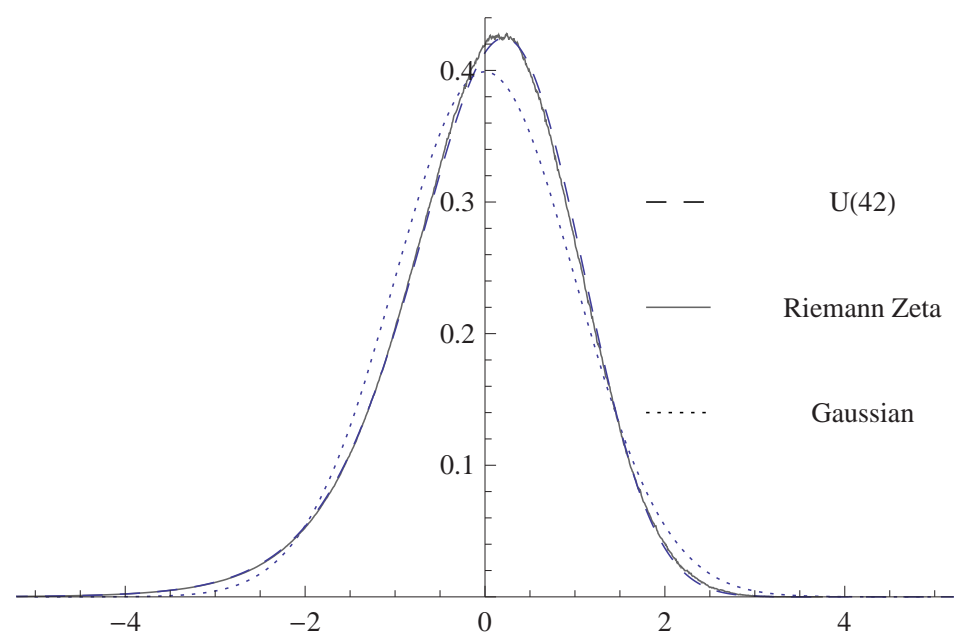

Figure 2: The value distribution for $\operatorname{Re} \log \Lambda_{A}$ with respect to matrices taken from $U(42)$, Odlyzko's data for the value distribution of $\operatorname{Re} \log \zeta(1 / 2+i t)$ near the $10^{20}$ th zero (taken from [58]), and the standard Gaussian, all scaled to have unit variance. (Taken from [53].)

unitary matrices. The natural function that has zeros at the eigenvalues of a matrix is the characteristic polynomial. The characteristic polynomial of a unitary matrix $A$ may be defined by

$$
\Lambda_{A}(s)=\operatorname{det}\left(I-A^{*} z\right)=\prod_{n=1}^{N}\left(1-s e^{-i \theta_{n}}\right),
$$

where $e^{i \theta_{1}}, \ldots, e^{i \theta_{N}}$ are the eigenvalues of $A$ and $A^{*}$ is its conjugate transpose. The following central limit theorem can be established for $\log \Lambda_{A}\left(e^{i \theta}\right)$ (see also $\left.[4,31]\right)$ :

Theorem 3.2 (Keating and Snaith, 2000 [53])

$$
\lim _{N \rightarrow \infty} \text { meas }\left\{A \in U(N): \frac{\log \Lambda_{A}\left(e^{i \theta}\right)}{\sqrt{\frac{1}{2} \log N}} \in B\right\}=\frac{1}{2 \pi} \iint_{B} e^{-\frac{1}{2}\left(x^{2}+y^{2}\right)} d x d y
$$

for rectangles $B \in \mathbb{C}$.

This theorem corresponds precisely to Selberg's for the value distribution of $\log \zeta(1 / 2+$ $i t$, suggesting that random matrix theory, in the limit as the matrix-size tends to infinity, can indeed model the value distribution of $\log \zeta(1 / 2+i t)$ as $t \rightarrow \infty$.

The question, though, is to explain the slow convergence to this limit seen in Odlyzko's numerics in Figure 2. We have seen that the limit $T \rightarrow \infty$ corresponds to the limit of matrix size $N \rightarrow \infty$. A natural way to relate $N$ and $T$ is to equate the density of zeros with the density of eigenvalues [53], giving, asymptotically,

$$
\frac{1}{2 \pi} \log \frac{T}{2 \pi}=\frac{N}{2 \pi} \text {. }
$$

We note this also has the effect, for large $T$ and $N$, of equating the variance $\frac{1}{2} \log \log T$ with $\frac{1}{2} \log N$. The $10^{20}$ th zero of the Riemann zeta function lies at height $T \sim 1.5202 \times 10^{19}$, 
yielding a value for $\mathrm{N}$ of about 42 . Thus on Figure 2 is also shown the distribution of Re $\log \Lambda_{A}(1)$, averaged over $A \in U(42)$. We see that characteristic polynomials of matrices of finite size are very effective at modelling the value distribution of the logarithm of the Riemann zeta function at finite height on the critical line. The moments of the distribution of the logarithm of the characteristic polynomial can be calculated. (For example, for the real part of the logarithm they can be calculated from the generating function that we will meet later, (4.5). For details see [53].) The lower order terms that contribute in the approach to the $N \rightarrow \infty$ limit describe the slow approach to the limit of the Riemann data in Figure 2.

In determining the value distribution of $\log \Lambda_{A}\left(e^{i \theta}\right)$ (e.g. as in Theorem 3.2), the averages were performed over matrices $A$ taken uniformly with respect to Haar measure on the unitary group $U(N)$. The value of $\theta$ chosen makes no difference as the unitary group is rotationally invariant. It is natural to ask how close this average is to an average with respect to $\theta$ when $A$ is fixed; that is, about ergodicity. It was proved in [44] that indeed the average is ergodic, in the sense that in the limit as $N \rightarrow \infty$, the average over $\theta$ equals that over $A$ for all but a set of matrices of zero measure.

As has been described above, the scaling of $\log \Lambda_{A}$ with respect to $\frac{1}{2} \log N$ leads to a central limit theorem. Different scalings, characterizing the large deviations of $\log \Lambda_{A}$, were also computed in [44], and shown to agree with numerical calculations and other results known to hold for the zeta function.

\section{Incorporating number theory}

At large, but finite, height on the critical line, the logarithm of the Riemann zeta function is modelled very effectively by finite size matrices. The arithmetic contribution to the distribution of the logarithm of the Riemann zeta function does not appear in the leading order term, and its effects are concentrated in the tails of the distribution so we do not see them in Figure 2 even for finite $T$. None the less, there is a contribution containing number theoretical information - random matrix theory alone cannot fully predict the behaviour for finite height on the critical line.

One sees this phenomenon clearly by plotting the two-point correlation of the unscaled zeros of the Riemann zeta function, and looking on a longer correlation scale than we did in Section 2. Now there appears a very different picture, see Figure 3. This plot is a histogram of the frequency of separation distances (between 0 and 40) occurring between pairs of zeros chosen from the lowest 100000 zeros of the Riemann zeta function.

We note in Figure 3 the dips in the data at values around 14, 21, etc. On the plot we have marked the positions of the lowest six Riemann zeros (approximately: 14.13, 21.02, $25.01,30.42,32.93,37.59)$. It is curious to note that pairs of Riemann zeros have a relatively small likelihood of being separated by a distance corresponding to the height of a low zero. This is something that cannot be predicted by random matrix theory, as random matrices do not contain information about specific positions of Riemann zeros. Clearly there is input of a number theoretic nature here. To understand how arithmetic and behaviour predicted by random matrix theory combine it is useful to follow the history of computing moments of the Riemann zeta function using random matrix theory. 


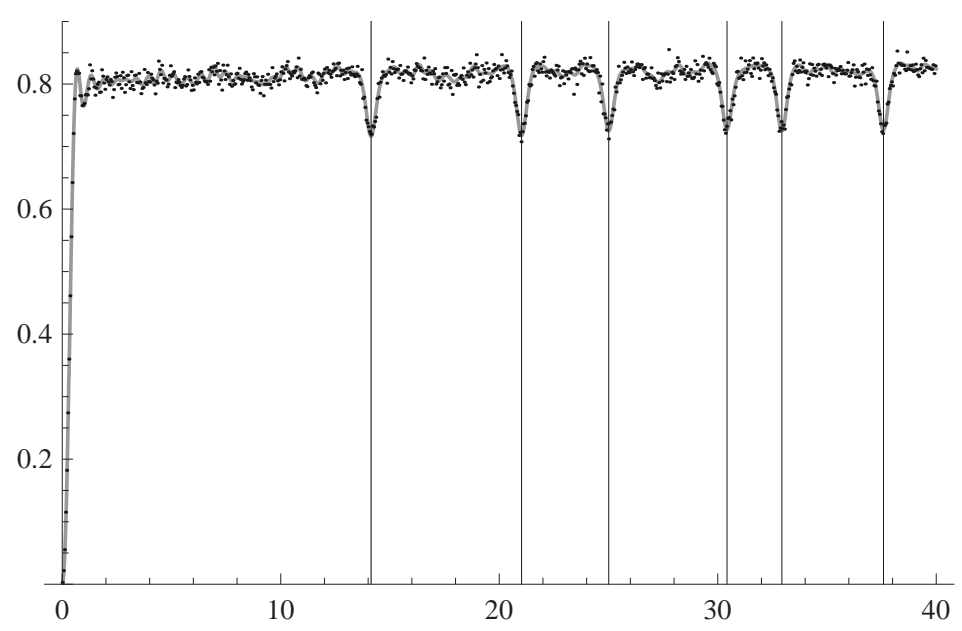

Figure 3: The dots represent the two-point correlation function of the raw Riemann zeros plotted using the first 100000 zeros, computed by A. Odlyzko. The solid grey line is the prediction from the ratios conjecture, given in (4.19). The positions of the lowest six Riemann zeros are marked, coinciding with the dips in the two-point statistic.

\subsection{Moments}

Number theorists believe the moments of the Riemann zeta function grow asymptotically like

$$
\frac{1}{T} \int_{0}^{T}|\zeta(1 / 2+i t)|^{2 \lambda} d t \sim a_{\lambda} \frac{g_{\lambda}}{\Gamma\left(1+\lambda^{2}\right)}(\log T)^{\lambda^{2}}
$$

for large $T$, where $a_{\lambda}$ is a product over primes

$$
a_{\lambda}=\prod_{p}\left(1-\frac{1}{p}\right)^{\lambda^{2}} \sum_{m=0}^{\infty}\left(\frac{\Gamma(m+\lambda)}{m ! \Gamma(\lambda)}\right)^{2} p^{-m},
$$

but $g_{\lambda}$ has remained elusive. Much work has been done on the subject of moments of the Riemann zeta function, for example $[1,2,23,24,25,33,39,40,41,42,48,49,59,60,61$, $65,63]$, but still $g_{1}=1$ [39] and $g_{2}=2$ [48] are the only proven values. Number theoretical methods have lead to conjectures for $g_{3}=42$ [24] and $g_{4}=24024$ [25] (and more recently for all moments [33]), but before random matrix techniques there was no viable conjecture for any further values.

To apply random matrix theory to this problem we once again use the characteristic polynomial $\Lambda_{A}(s)$ to model the Riemann zeta function. The analogous quantity to (4.1) is

$$
M_{N}(\lambda)=\int_{U(N)}\left|\Lambda_{A}(1)\right|^{2 \lambda} d A_{\text {Haar }},
$$

where the integration is, as before, with respect to Haar measure. We average the characteristic polynomial at $s=1$ because the unitary group is rotationally invariant and so it makes no difference at what position on the unit circle we evaluate the average. Weyl's integration 
formula [68] allows us to write Haar measure on $U(N)$ in terms of the eigenangles, giving

$$
\begin{aligned}
& \int_{U(N)}\left|\Lambda_{A}(1)\right|^{2 \lambda} d A_{\text {Haar }} \\
& \quad=\frac{1}{(2 \pi)^{N} N !} \int_{0}^{2 \pi} \cdots \int_{0}^{2 \pi} \prod_{1 \leq j<m \leq N}\left|e^{i \theta_{j}}-e^{i \theta_{m}}\right|^{2}\left|\prod_{n=1}^{N}\left(1-e^{-i \theta_{n}}\right)\right|^{2 \lambda} d \theta_{1} \cdots d \theta_{N} .
\end{aligned}
$$

This $N$-dimensional integral may then be computed by relating it to an integral evaluated by Selberg (see [54]) for $-1 / 2<\operatorname{Re}(\lambda)$, giving

$$
\begin{aligned}
M_{N}(\lambda) & =\prod_{j=1}^{N} \frac{\Gamma(j) \Gamma(j+2 \lambda)}{(\Gamma(j+\lambda))^{2}} \\
& \sim f(\lambda) N^{\lambda^{2}}
\end{aligned}
$$

where the final line is the asymptotic for large $N$. For integer $\lambda=k$ the leading order coefficient is

$$
f(k)=\prod_{j=0}^{k-1} \frac{j !}{(j+k) !},
$$

and for arbitrary $\lambda$ the general expression is

$$
f(\lambda)=\frac{(G(1+\lambda))^{2}}{G(1+2 \lambda)}
$$

Here we use the Barnes double gamma function [5] in the expression for $f(\lambda)$. It is related to the gamma function, having the property

$$
\begin{aligned}
G(1) & =1, \\
G(z+1) & =\Gamma(z) G(z) .
\end{aligned}
$$

Note that, in reference to Section 3, all information about the value distribution of $\operatorname{Re} \log \Lambda$ on the unit circle is contained within (4.5): moments may be computed in terms of the derivatives of $M_{N}(\lambda)$ at $\lambda=0$, and the value distribution itself is the fourier transform of $M_{N}(i y)$. Information about the value distribution of $\operatorname{Im} \log \Lambda$, and the joint value distribution of the real and imaginary parts of $\log \Lambda$ may be computed in a similar way [53].

It is conjectured [53] that

$$
f(\lambda)=\frac{g_{\lambda}}{\Gamma\left(1+\lambda^{2}\right)}
$$

or equivalently

$$
\begin{aligned}
\lim _{T \rightarrow \infty}(\log T)^{-\lambda^{2}} \frac{1}{T} \int_{0}^{T}|\zeta(1 / 2+i t)|^{2 \lambda} d t \\
\quad=a_{\lambda} \lim _{N \rightarrow \infty} N^{-\lambda^{2}} \int_{U(N)}\left|\Lambda_{A}(1)\right|^{2 \lambda} d A_{\text {Haar }}
\end{aligned}
$$

We see that here again $N$ is playing the role of $\log T$, and also that here even in the leading order coefficient arithmetic information contributes, in the form of $a_{\lambda}$. This conjecture 
agrees with the previously known and (independently) conjectured results for $g_{k}$ for $k=$ $1,2,3,4$.

It is clear from this conjecture that random matrix theory alone cannot always predict the behaviour of quantities involving the Riemann zeta function, even in the limit $T \rightarrow \infty$. The key is to ascertain how to combine random matrix behaviour with the intricate number theoretical aspects that are specific to the Riemann zeta function.

\subsection{Shifted moments}

In the previous section we considered only the leading order (in large $T$ ) of the moments of the Riemann zeta function. It turns out that an effective way to understand the lower order terms is to consider instead "shifted" moments. These are quantities of the form

$$
\begin{aligned}
\frac{1}{T} \int_{0}^{T} \zeta\left(1 / 2+i t+\alpha_{1}\right) \cdots \zeta\left(1 / 2+i t+\alpha_{k}\right) \\
\quad \times \zeta\left(1 / 2-i t-\alpha_{k+1}\right) \cdots \zeta\left(1 / 2-i t-\alpha_{2 k}\right) d t
\end{aligned}
$$

Conjectures for these shifted moments were developed by Conrey, Farmer, Keating, Rubinstein and Snaith, where they also propose a "recipe" for conjecturing such moments in other situations, for example, for averages over families of $L$-functions. See [19] for the precise form of these conjectures. The important point is that these conjectures, which derive from a highly non-rigorous but nonetheless number theoretical argument, have an identical structure to the analogous moment in random matrix theory [18] (see also [11]):

$$
\int_{U(N)} \Lambda_{A}\left(e^{-\alpha_{1}}\right) \cdots \Lambda_{A}\left(e^{-\alpha_{k}}\right) \Lambda_{A^{*}}\left(e^{\alpha_{k+1}}\right) \Lambda_{A^{*}}\left(e^{\alpha_{2 k}}\right) d A_{\text {Haar }}
$$

The major features of the formulae for (4.12) and (4.13) are the same, but the conjectural result for (4.12) it is embellished with arithmetic details that incorporate specific information about the Riemann zeta function. We will see an example of this in (4.17) and (4.18).

Once a conjecture for (4.12) has been accepted, setting the $\alpha$ 's to zero reduces the expression to a formula for the moments (4.1), and this leads to a conjecture that goes deeper than just the leading order term:

$$
\int_{A}^{B}\left|\zeta\left(\frac{1}{2}+i t\right)\right|^{2 k} d t=\int_{A}^{B} P_{k}\left(\log \frac{t}{2 \pi}\right)\left(1+O\left(t^{-\frac{1}{2}+\epsilon}\right)\right) d t
$$

where $P_{k}(x)$ is a polynomial of degree $k^{2}$. As an example, the 4 th degree polynomial 
associated with the moment when $k=2$ is

$$
\begin{aligned}
& P_{2}(x)=\frac{1}{2 \pi^{2}} x^{4}+\frac{8}{\pi^{4}}\left(\gamma \pi^{2}-3 \zeta^{\prime}(2)\right) x^{3}+ \\
& \frac{6}{\pi^{6}}\left(-48 \gamma \zeta^{\prime}(2) \pi^{2}-12 \zeta^{\prime \prime}(2) \pi^{2}+7 \gamma^{2} \pi^{4}+144 \zeta^{\prime}(2)^{2}-2 \gamma_{1} \pi^{4}\right) x^{2}+ \\
& \frac{12}{\pi^{8}}\left(6 \gamma^{3} \pi^{6}-84 \gamma^{2} \zeta^{\prime}(2) \pi^{4}+24 \gamma_{1} \zeta^{\prime}(2) \pi^{4}-1728 \zeta^{\prime}(2)^{3}+576 \gamma \zeta^{\prime}(2)^{2} \pi^{2}+\right. \\
& \left.288 \zeta^{\prime}(2) \zeta^{\prime \prime}(2) \pi^{2}-8 \zeta^{\prime \prime \prime}(2) \pi^{4}-10 \gamma_{1} \gamma \pi^{6}-\gamma_{2} \pi^{6}-48 \gamma \zeta^{\prime \prime}(2) \pi^{4}\right) x+ \\
& \frac{4}{\pi^{10}}\left(-12 \zeta^{\prime \prime \prime \prime}(2) \pi^{6}+36 \gamma_{2} \zeta^{\prime}(2) \pi^{6}+9 \gamma^{4} \pi^{8}+21 \gamma_{1}^{2} \pi^{8}+432 \zeta^{\prime \prime}(2)^{2} \pi^{4}+\right. \\
& 3456 \gamma \zeta^{\prime}(2) \zeta^{\prime \prime}(2) \pi^{4}+3024 \gamma^{2} \zeta^{\prime}(2)^{2} \pi^{4}-36 \gamma^{2} \gamma_{1} \pi^{8}-252 \gamma^{2} \zeta^{\prime \prime}(2) \pi^{6}+ \\
& 3 \gamma \gamma_{2} \pi^{8}+72 \gamma_{1} \zeta^{\prime \prime}(2) \pi^{6}+360 \gamma_{1} \gamma \zeta^{\prime}(2) \pi^{6}-216 \gamma^{3} \zeta^{\prime}(2) \pi^{6}-864 \gamma_{1} \zeta^{\prime}(2)^{2} \pi^{4}+ \\
& 5 \gamma_{3} \pi^{8}+576 \zeta^{\prime}(2) \zeta^{\prime \prime \prime}(2) \pi^{4}-20736 \gamma \zeta^{\prime}(2)^{3} \pi^{2}-15552 \zeta^{\prime \prime}(2) \zeta^{\prime}(2)^{2} \pi^{2}- \\
& \left.96 \gamma \zeta^{\prime \prime \prime}(2) \pi^{6}+62208 \zeta^{\prime}(2)^{4}\right)
\end{aligned}
$$

Whereas with just the leading order term numerical testing of the conjecture was not particularly convincing, the more accurate formula (4.14), derived from the shifted moments, compares very well with numerics, as seen in Table 1 (taken from [19]). Note in particular the excellent agreement between numerics and theory in the final line of the table where a large interval of integration was used.

\subsection{Ratios of the Riemann zeta function}

A further generalization was made by Conrey, Farmer and Zirnbauer [21] to averages of ratios of the Riemann zeta function:

$$
\frac{1}{T} \int_{0}^{T} \frac{\prod_{k=1}^{K} \zeta\left(1 / 2+i t+\alpha_{k}\right) \prod_{\ell=K+1}^{K+L} \zeta\left(1 / 2-i t-\alpha_{\ell}\right)}{\prod_{q=1}^{Q} \zeta\left(1 / 2+i t+\gamma_{q}\right) \prod_{r=1}^{R} \zeta\left(1 / 2-i t+\delta_{r}\right)} d t .
$$

They develop a similar recipe for generating conjectures for quantities of this type. Once again the structure mirrors that of the analogous random matrix quantity (see [20, 14, 22] for this calculation).

As an example we consider

Conjecture 4.1 (Conrey, Farmer, Zirnbauer, $2008[21]$ ) Let $-1 / 4<\operatorname{Re}(\alpha), \operatorname{Re}(\beta)<$ $1 / 4,1 / \log T \ll \operatorname{Re}(\gamma), \operatorname{Re}(\delta)<1 / 4$ and $\operatorname{Im}(\alpha), \operatorname{Im}(\beta), \operatorname{Im}(\gamma), \operatorname{Im}(\delta) \ll_{\epsilon} T^{1-\epsilon}$ for every $\epsilon>0$. Then with $s=1 / 2+i$,

$$
\begin{gathered}
\int_{0}^{T} \frac{\zeta(s+\alpha) \zeta(1-s+\beta)}{\zeta(s+\gamma) \zeta(1-s+\delta)} d t=\int_{0}^{T}\left(\frac{\zeta(1+\alpha+\beta) \zeta(1+\gamma+\delta)}{\zeta(1+\alpha+\delta) \zeta(1+\beta+\gamma)} A_{\zeta}(\alpha, \beta ; \gamma, \delta)\right. \\
\left.+e^{-\log \frac{t}{2 \pi}(\alpha+\beta)} \frac{\zeta(1-\alpha-\beta) \zeta(1+\gamma+\delta)}{\zeta(1-\beta+\delta) \zeta(1-\alpha+\gamma)} A_{\zeta}(-\beta,-\alpha ; \gamma, \delta)\right) d t \\
+O\left(T^{1 / 2+\epsilon}\right) .
\end{gathered}
$$

We compare this to the following: 


\begin{tabular}{|c|c|c|c|}
\hline$[A, B]$ & conjecture $(4.14)$ & numerical average & ratio \\
\hline$[0,50000]$ & 7236872972.7 & 7231005642.3 & .999189 \\
{$[50000,100000]$} & 15696470555.3 & 15723919113.6 & 1.001749 \\
{$[100000,150000]$} & 21568672884.1 & 21536840937.9 & .998524 \\
{$[150000,200000]$} & 26381397608.2 & 26246250354.1 & .994877 \\
{$[200000,250000]$} & 30556177136.5 & 30692229217.8 & 1.004453 \\
{$[250000,300000]$} & 34290291841.0 & 34414329738.9 & 1.003617 \\
{$[300000,350000]$} & 37695829854.3 & 37683495193.0 & .999673 \\
{$[350000,400000]$} & 40843941365.7 & 40566252008.5 & .993201 \\
{$[400000,450000]$} & 43783216365.2 & 43907511751.1 & 1.002839 \\
{$[450000,500000]$} & 46548617846.7 & 46531247056.9 & .999627 \\
{$[500000,550000]$} & 49166313161.9 & 49136264678.2 & .999389 \\
{$[550000,600000]$} & 51656498739.2 & 51744796875.0 & 1.001709 \\
{$[600000,650000]$} & 54035153255.1 & 53962410634.2 & .998654 \\
{$[650000,700000]$} & 56315178564.8 & 56541799179.3 & 1.004024 \\
{$[700000,750000]$} & 58507171421.6 & 58365383245.2 & .997577 \\
{$[750000,800000]$} & 60619962488.2 & 60870809317.1 & 1.004138 \\
{$[800000,850000]$} & 62661003164.6 & 62765220708.6 & 1.001663 \\
{$[850000,900000]$} & 64636649728.0 & 64227164326.1 & .993665 \\
{$[900000,950000]$} & 66552376294.2 & 65994874052.2 & .991623 \\
{$[950000,1000000]$} & 68412937271.4 & 68961125079.8 & 1.008013 \\
{$[1000000,1050000]$} & 70222493232.7 & 70233393177.0 & 1.000155 \\
{$[1050000,1100000]$} & 71984709805.4 & 72919426905.7 & 1.012985 \\
{$[1100000,1150000]$} & 73702836332.4 & 72567024812.4 & .984589 \\
{$[1150000,1200000]$} & 75379769148.4 & 76267763314.7 & 1.011780 \\
{$[1200000,1250000]$} & 77018102997.5 & 76750297112.6 & .996523 \\
{$[1250000,1300000]$} & 78620173202.6 & 78315210623.9 & .996121 \\
{$[1300000,1350000]$} & 80188090542.5 & 80320710380.9 & 1.001654 \\
{$[1350000,1400000]$} & 81723770322.2 & 80767881132.6 & .988303 \\
{$[1400000,1450000]$} & 83228956776.3 & 83782957374.3 & 1.006656 \\
{$[0,2350000]$} & 3317437762612.4 & 3317496016044.9 & 1.000017 \\
\hline
\end{tabular}

Table 1: Sixth moment of $\zeta$ versus the conjecture 4.14. The 'numerical average' column, i.e. integrals involving $\zeta$, were computed using Mathematica.

Theorem 4.2 (Conrey, Farmer, Zirnbauer $[20]$ ) For $\operatorname{Re}(\gamma), \operatorname{Re}(\delta)>0$,

$$
\begin{aligned}
& \int_{U(N)} \frac{\Lambda_{A}\left(e^{-\alpha}\right) \Lambda_{A^{*}}\left(e^{-\beta}\right)}{\Lambda_{A}\left(e^{-\gamma}\right) \Lambda_{A^{*}}\left(e^{-\delta}\right)} d A_{\mathrm{Haar}}=\frac{z(\alpha+\beta) z(\gamma+\delta)}{z(\alpha+\delta) z(\beta+\gamma)} \\
& +e^{-N(\alpha+\beta)} \frac{z(-\beta-\alpha) z(\gamma+\delta)}{z(-\beta+\delta) z(-\alpha+\gamma)}
\end{aligned}
$$

with $z(x)=\left(1-e^{-x}\right)^{-1}$.

The interesting point is that $z(x)$ has a pole at $x=0$ with residue 1 , exactly as does $\zeta(1+x)$. Comparing (4.17) and (4.18) in this light shows that the structure of the two formulae are identical: $z(\alpha+\beta)$ takes the place of $\zeta(1+\alpha+\beta)$, etc. Remembering that we relate $N$ to $\log \frac{t}{2 \pi}$, we see clearly that the formulae mirror each other remarkably well. $A_{\zeta}(\alpha, \beta ; \gamma, \delta)$ is 
a product over primes, and $\zeta(1+x)$ contains arithmetic information that $z(x)$ lacks, but the overall behaviour of these expressions is dominated by their polar structure, and this is identical in the two cases. This gives strong support to the conjecture in the number theory case. Even apart from the connection with number theory, averages of ratios of characteristic polynomials have been the subject of work in random matrix theory (see for example $[3,9,14,37])$.

Upon accepting Conjecture 4.1 we can now fully understand the two-point correlation function in Figure 3. By taking a logarithmic derivative of (4.17) with respect to the variables $\alpha$ and $\beta$, subsequently setting $\gamma=\alpha$ and $\delta=\beta$ and performing a double integration, integrated against a suitable test function around a contour that encloses the zeros with $0<\gamma_{j} \leq T$, we obtain a detailed expression for the two-point correlation function [28].

Theorem 4.3 Assuming Conjecture 4.1, and with $f$ a suitable, even, test function, we have

$$
\begin{gathered}
\sum_{0 \leq \gamma_{j}, \gamma_{k} \leq T} f\left(\gamma_{j}-\gamma_{k}\right)=\frac{1}{(2 \pi)^{2}} \int_{0}^{T}\left(2 \pi f(0) \log \frac{t}{2 \pi}+\int_{-T}^{T} f(r)\left[\log ^{2} \frac{t}{2 \pi}+2\left(\left(\frac{\zeta^{\prime}}{\zeta}\right)^{\prime}(1+i r)\right.\right.\right. \\
\left.\left.\left.+\left(\frac{t}{2 \pi}\right)^{-i r} \zeta(1-i r) \zeta(1+i r) A(i r)-B(i r)\right)\right] d r\right) d t+O\left(T^{1 / 2+\epsilon}\right)
\end{gathered}
$$

here the integral is to be regarded as a principal value near $r=0$,

$$
A(\eta)=\prod_{p} \frac{\left(1-\frac{1}{p^{1+\eta}}\right)\left(1-\frac{2}{p}+\frac{1}{p^{1+\eta}}\right)}{\left(1-\frac{1}{p}\right)^{2}},
$$

and

$$
B(\eta)=\sum_{p}\left(\frac{\log p}{\left(p^{1+\eta}-1\right)}\right)^{2} .
$$

To compare with the numerical two-point correlation function we plot the quantity in square brackets as the solid curve in Figure 3. It is the term containing $\left(\frac{\zeta^{\prime}}{\zeta}\right)^{\prime}(1+i r)$, a derivative of the zeta function on the line with real part equal to 1 , that feels the influence of the low Riemann zeros and accounts for the dips at these positions on the figure. The expression (4.19) was first derived by Bogomolny and Keating [7] by a different method, which could be extended to treat the higher correlation functions as well. The higher correlation functions have been obtained from the ratios conjectures in [30, 29].

\subsection{The hybrid formula and extreme values}

In Section 4.1, it is noticeable that the leading order coefficient of the moments of the Riemann zeta function splits into a product of arithmetic information $a_{\lambda}$ and a factor determined by random matrix theory. This inspired a "hybrid" formula, developed by Gonek, Hughes and Keating [38]. The zeta function can be written as an Euler product over primes, or as a Hadamard product over its zeros. Combining these two forms, using an explicit formula connecting the zeros and the primes, Gonek, Hughes and Keating derive a hybrid product: 
Theorem 4.4 (Gonek, Hughes and Keating, 2007 [38]) For $s=\sigma+i t$ with $0 \leq \sigma$ and $|t| \geq 2$, let $X \geq 2$ be a real parameter, and let $K$ be any fixed positive integer. Let $f(x)$ be a nonnegative $C^{\infty}$-function of mass one supported on $[0,1]$, and set $u(x)=X f(X \log (x / e)+$ $1) / x$. Thus $u(x)$ is a function of mass one supported on $\left[e^{1-1 / X}, e\right]$. Set

$$
U(z)=\int_{0}^{\infty} u(x) E_{1}(z \log x) d x
$$

where $E_{1}(z)$ is the exponential integral $\int_{z}^{\infty} e^{-w} / w d w$. Then

$$
\zeta(s)=P_{X}(s) Z_{X}(s)\left(1+O\left(\frac{X^{K+2}}{(|s| \log X)^{K}}\right)+O\left(X^{-\sigma} \log X\right)\right)
$$

where

$$
P_{X}(s)=\exp \left(\sum_{n \leq X} \frac{\Lambda(n)}{n^{s} \log n}\right)
$$

with

$$
\Lambda(n)=\left\{\begin{array}{cc}
\log p & \text { if } n \text { is a power of a prime } p \\
0 & \text { else }
\end{array}\right.
$$

and

$$
Z_{X}(s)=\exp \left(-\sum_{\rho_{n}} U\left(\left(s-\rho_{n}\right) \log X\right) .\right.
$$

The constants implied by the $O$-terms depend only on $f$ and $K$.

We see that the parameter $X$ acts to moderate the balance between the product over zeros and the product over primes: $U$ limits the sum over zeros to those near $s$ (on a scale of $1 / \log X)$, and the product over primes goes up to $X$.

This hybrid product is used in [38] to reconsider the moments of the Riemann zeta function. The authors make progress by conjecturing that upon averaging $\zeta(\sigma+i t)$ over $t$, the averages of $P_{X}$ and $Z_{X}$ are independent (something they prove in particular cases).

Very interestingly, the hybrid product also led to a conjecture concerning the extreme values achieved by the Riemann zeta function on the critical line. Taking $t>0$, the Lindelöf Hypothesis asserts that for every $\epsilon>0, \zeta(1 / 2+i t)=O\left(t^{\epsilon}\right)$. With the assumption of the Riemann hypothesis it has been shown [15] that

$$
\zeta\left(\frac{1}{2}+i t\right)=O\left(\exp \left(\frac{\log 2}{2} \frac{\log t}{\log \log t}\right)\right) .
$$

On the other hand [64], there are arbitrarily large $t$ such that

$$
\left|\zeta\left(\frac{1}{2}+i t\right)\right| \geq \exp \left((1+o(1)) \sqrt{\frac{\log t}{\log \log t}}\right) .
$$

Farmer, Gonek and Hughes use the hybrid formula to present a case for the following:

Conjecture 4.5 (Farmer, Gonek and Hughes, 2007 [36])

$$
\zeta\left(\frac{1}{2}+i t\right)=O\left(\exp \left(\left(\frac{1}{\sqrt{2}}+\epsilon\right) \sqrt{\log t \log \log t}\right)\right)
$$

for all $\epsilon>0$ and for no $\epsilon<0$. 


\section{Probabilistic interpretation of moments of characteristic polynomials}

There have recently been developments on a new probabilistic model for characteristic polynomials. Bourgade, Hughes, Nikeghbali and Yor [10] have shown that averages of characteristic polynomials behave in the same way as products of independent beta variables. We state their theorem here and follow it with some explanation and applications.

Theorem 5.1 Let $V_{N} \in U(N)$ be distributed with the Haar measure $\mu_{U(N)}$. Then for all $\theta \in \mathbb{R}$

$$
\operatorname{det}\left(I-e^{i \theta} V_{N}\right) \stackrel{\text { law }}{=} \prod_{k=1}^{N}\left(1+e^{i \theta_{k}} \sqrt{\beta_{1, k-1}}\right)
$$

with $\theta_{1}, \ldots, \theta_{n}, \beta_{1,0}, \ldots, \beta_{1, n-1}$ independent random variables, the $\theta_{k}$ 's uniformly distributed on $[0,2 \pi]$, and the $\beta_{1, j}$ 's $(0 \leq j \leq N-1)$ being beta-distributed with parameters 1 and $j$. (By convention, $\beta_{1,0}$ is the Dirac distribution on 1.)

The theorem states that the characteristic polynomial of a Haar distributed unitary matrix is equal in law to a product of independent random variables. Equality in law for two random variables $X$ and $Y$ means that for suitable test functions from the domain of the random variable to $\mathbb{R}$,

$$
\mathbb{E}[f(X)]=\mathbb{E}[f(Y)]
$$

where $\mathbb{E}$ is the expectation value. The probability density of a beta-distributed random variable, defined on $[0,1]$, with parameters $\alpha$ and $\beta$ is

$$
\frac{\Gamma(\alpha+\beta)}{\Gamma(\alpha) \Gamma(\beta)} x^{\alpha-1}(1-x)^{\beta-1}
$$

From the above theorem, with a bit of work on expectation values of an individual beta-distributed random variable, the authors rederive (4.5). They also revisit the distribution of the logarithm of the characteristic polynomial of a random $U(N)$ matrix, the real and imaginary parts of which are known to be independently Gaussian distributed in the limit of large matrix size, as we saw in Section 3. The Gaussian behaviour in the limit follows naturally from the probabilistic model as the logarithm of the characteristic polynomial can be decomposed into sums of independent random variables and then classical central limit theorems can be applied, and in addition the authors of [10] obtain new results in the form of estimates on the rate of convergence to the Gaussian limit.

\section{Conclusions and extensions}

We see that random matrix theory is a useful tool in exploring the statistical behaviour of values of the Riemann zeta function and the positions of its zeros. In this article we have confined the discussion to the Riemann zeta function, but there also exist many other $L$ functions with similar properties: a representation as a Dirichlet series, an Euler product, a functional equation and a Generalized Riemann Hypothesis. Any random matrix calculation applied to the Riemann zeta function could equally be applied to such an $L$-function (see, for example, [62]). In addition, Katz and Sarnak [50, 51] proposed that collections of $L$ functions gathered into natural families also show random matrix statistics, and much work 
has been done using random matrix theory to calculate moments and zero statistics in this case, too, leading to surprising new applications $[12,13,16,26,27,28,32,45,46,47,52$, $55,56,66]$.

\section{References}

[1] F.V. Atkinson, The mean value of the zeta-function on the critical line, Quart. J. Math. Oxford Ser., 10:122-128, 1939.

[2] F.V. Atkinson, The mean value of the zeta-function on the critical line, Proc. London Math. Soc. (2), 47:174-200, 1941.

[3] J. Baik, P. Deift, and E. Strahof, Products and ratios of characteristic polynomials of random Hermitian matrices, J. Math. Phys., 44(8):3657-70, 2003.

[4] T.H. Baker and P.J. Forrester, Finite- $N$ fluctuation formulas for random matrices, $J$. Stat. Phys., 88:1371-1385, 1997.

[5] E.W. Barnes, The theory of the G-function, Q. J. Math., 31:264-314, 1900.

[6] E.B. Bogomolny and J.P. Keating, Random matrix theory and the Riemann zeros I: three- and four-point correlations, Nonlinearity, 8:1115-1131, 1995.

[7] E.B. Bogomolny and J.P. Keating, Gutzwiller's trace formula and spectral statistics: beyond the diagonal approximation, Phys. Rev. Lett., 77(8):1472-1475, 1996.

[8] E.B. Bogomolny and J.P. Keating, Random matrix theory and the Riemann zeros II:n-point correlations, Nonlinearity, 9:911-935, 1996.

[9] A. Borodin and E. Strahov, Averages of characteristic polynomials in random matrix theory, Comm. Pure Appl. Math., 59(2):161-253, 2006.

[10] P. Bourgade, C.P. Hughes, A. Nikeghbali, and M. Yor, The characteristic polynomial of a random unitary matrix: A probabilistic approach, Duke Math. J., 145(1):45-69, 2008.

[11] E. Brézin and S. Hikami, Characteristic polynomials of random matrices, Comm. Math. Phys., 214:111-135, 2000, arXiv:math-ph/9910005.

[12] H. Bui and J.P. Keating, On the mean values of Dirichlet $L$-functions, Proc. London Math. Soc., 95(3):273-298, 2007.

[13] H. Bui and J.P. Keating, On the mean values of $L$-functions in orthogonal and symplectic families, Proc. London Math. Soc., 96(3):335-366, 2008.

[14] D. Bump and A. Gamburd, On the averages of characteristic polynomials from classical groups, Comm. Math. Phys., 265(1):227-274, 2006, arXiv:math-ph/0502043.

[15] V. Chandee and K. Soundararajan, Bounding $|\zeta(1 / 2+i t)|$ on the Riemann hypothesis, preprint, arXiv:0908.2008. 
[16] J.B. Conrey, Families of $L$-functions and 1-level densities, In Recent perspectives on random matrix theory and number theory, LMS Lecture Note Series 322, pages 225-49. Cambridge University Press, Cambridge, 2005.

[17] J.B. Conrey, Notes on eigenvalue distributions for the classical compact groups, In Recent perspectives on random matrix theory and number theory, LMS Lecture Note Series 322, pages 111-45. Cambridge University Press, Cambridge, 2005.

[18] J.B. Conrey, D.W. Farmer, J.P. Keating, M.O. Rubinstein, and N.C. Snaith, Autocorrelation of random matrix polynomials, Comm. Math. Phys., 237(3):365-395, 2003, arXiv:math-ph/0208007.

[19] J.B. Conrey, D.W. Farmer, J.P. Keating, M.O. Rubinstein, and N.C. Snaith, Integral moments of L-functions, Proc. London Math. Soc., 91(1):33-104, 2005, arXiv:math.nt/0206018.

[20] J.B. Conrey, D.W. Farmer, and M.R. Zirnbauer, Howe pairs, supersymmetry, and ratios of random characteristic polynomials for the unitary groups $U(N)$, preprint, arXiv:math-ph/0511024.

[21] J.B. Conrey, D.W. Farmer, and M.R. Zirnbauer, Autocorrelation of ratios of $L$ functions, Comm. Number Theory and Physics, 2(3):593-636, 2008, arXiv:0711.0718.

[22] J.B. Conrey, P.J. Forrester, and N.C. Snaith, Averages of ratios of characteristic polynomials for the compact classical groups, Int. Math. Res. Notices, 7:397-431, 2005 .

[23] J.B. Conrey and A. Ghosh, On mean values of the zeta-function, Mathematika, 31:159161, 1984.

[24] J.B. Conrey and A. Ghosh, Mean values of the zeta-function, iii, Proceedings of the Amalfi Conference on Analytic Number Theory, Università di Salerno, 1992.

[25] J.B. Conrey and S.M. Gonek, High moments of the Riemann zeta-function, Duke Math. J., 107:577-604, 2001.

[26] J.B. Conrey, J.P. Keating, M.O. Rubinstein, and N.C. Snaith, On the frequency of vanishing of quadratic twists of modular L-functions, In Number Theory for the Millennium I: Proceedings of the Millennial Conference on Number Theory; editor, M.A. Bennett et al., pages 301-315. A K Peters, Ltd, Natick, 2002, arXiv:math.nt/0012043.

[27] J.B. Conrey, A. Pokharel, M.O. Rubinstein, and M. Watkins, Secondary terms in the number of vanishings of quadratic twists of elliptic curve $L$-functions, In Ranks of Elliptic Curves and Random Matrix Theory, LMS lecture note series 341, pages 215-232. Cambridge University Press, Cambridge, 2007, arXiv:math.NT/0509059.

[28] J.B. Conrey and N.C. Snaith, Applications of the $L$-functions ratios conjectures, Proc. London Math. Soc., 94(3):594-646, 2007, arXiv:math.NT/0509480.

[29] J.B. Conrey and N.C. Snaith, Correlations of eigenvalues and Riemann zeros, Comm. Number Theory and Phyics, 2(3):477-536, 2008, arXiv:0803.2795. 
[30] J.B. Conrey and N.C. Snaith, Triple correlation of the Riemann zeros, Journal de Théorie des Nombres de Bordeaux, 20:61-106, 2008, arXiv:math/0610495.

[31] O. Costin and J.L. Lebowitz, Gaussian fluctuation in random matrices, Phys. Rev. Lett., 75(1):69-72, 1995.

[32] C. David, J. Fearnley, and H. Kisilevsky, On the vanishing of twisted $L$-functions of elliptic curves, Experiment. Math., 13(2):185-98, 2004.

[33] A. Diaconu, D. Goldfeld, and J. Hoffstein, Multiple Dirichlet series and moments of zeta- and L-functions, Compos. Math., 139(3):297-360, 2003, arXiv:math.nt/0110092.

[34] F.J. Dyson, Statistical theory of the energy levels of complex systems, i, ii and iii, $J$. Math. Phys., 3:140-175, 1962.

[35] H.M. Edwards, Riemann's Zeta Function, Dover Publications, Inc., New York, 1974.

[36] D.W. Farmer, S. M. Gonek, and C.P. Hughes, The maximum size of $L$-functions, Journal fur die Reine und angewandte mathematik, 609:215-36, 2007.

[37] Y.V. Fyodorov and E. Strahov, An exact formula for general spectral correlation function of random Hermitian matrices, J. Phys. A: Math. Gen., 36(12):3203-13, 2003, arXiv:math-ph/0204051.

[38] S.M. Gonek, C.P. Hughes, and J.P. Keating, A hybrid Euler-Hadamard product formula for the Riemann zeta function, Duke Math. J., 136(3):507-549, 2007.

[39] G.H. Hardy and J.E. Littlewood, Contributions to the theory of the Riemann zetafunction and the theory of the distribution of primes, Acta Math., 41:119-196, 1918.

[40] D.R. Heath-Brown, The fourth power moment of the Riemann zeta function, Proc. London Math. Soc. (3), 38:385-422, 1979.

[41] D.R. Heath-Brown, Fractional moments of the Riemann zeta-function, J.London Math. Soc. (2), 24:65-78, 1981.

[42] D.R. Heath-Brown, Fractional moments of the Riemann zeta-function, ii, Quart. J. Math. Oxford Ser. (2), 44:185-197, 1993.

[43] D.A. Hejhal, On the triple correlation of zeros of the zeta function, Inter. Math. Res. Notices, 7:293-302, 1994.

[44] C.P. Hughes, J.P. Keating, and N. O'Connell, On the characteristic polynomial of a random unitary matrix, Comm. Math. Phys., 220(2):429-451, 2001.

[45] C.P. Hughes and S.J. Miller, Low-lying zeros of $L$-functions with orthogonal symmetry, Duke Math. J., 136(1):115-172, 2007.

[46] C.P. Hughes and Z. Rudnick, Linear statistics of low-lying zeros of $L$-functions, Q. $J$. Math., 54(3):309-333, 2003, arXiv:math.nt/0208230.

[47] D.K. Huynh, J.P. Keating, and N.C. Snaith, Lower order terms for the one-level density of elliptic curve $L$-functions, J. Number Theory, 129:2883-2902, 2009, arXiv:0811.2304. 
[48] A.E. Ingham, Mean-value theorems in the theory of the Riemann zeta-function, Proc. London Math. Soc. (2), 27:273-300, 1926.

[49] M. Jutila, On the value distribution of the zeta-function on the critical line, Bull. London Math. Soc., 15:513-518, 1983.

[50] N.M. Katz and P. Sarnak, Random Matrices, Frobenius Eigenvalues and Monodromy, American Mathematical Society Colloquium Publications, 45. American Mathematical Society, Providence, Rhode Island, 1999.

[51] N.M. Katz and P. Sarnak, Zeros of zeta functions and symmetry, Bull. Amer. Math. Soc., 36:1-26, 1999.

[52] J.P. Keating and N.C. Snaith, Random matrix theory and $L$-functions at $s=1 / 2$, Comm. Math. Phys, 214:91-110, 2000.

[53] J.P. Keating and N.C. Snaith, Random matrix theory and $\zeta(1 / 2+i t)$, Comm. Math. Phys., 214:57-89, 2000.

[54] M.L. Mehta, Random Matrices, Academic Press, London, second edition, 1991.

[55] S.J. Miller, A symplectic test of the $L$-functions ratios conjecture, Int. Math. Res. Notices, 2008, article ID rnm 146, arXiv:0704.0927.

[56] S.J. Miller, An orthogonal test of the $L$-functions ratios conjecture, Proc. London Math. Soc., 99:484-520, 2009, arXiv:0805.4208.

[57] H.L. Montgomery, The pair correlation of the zeta function, Proc. Symp. Pure Math, 24:181-93, 1973.

[58] A.M. Odlyzko, The $10^{20}$ th zero of the Riemann zeta function and 70 million of its neighbors, preprint, 1989, http://www.dtc.umn.edu/ ${ }^{\circ}$ odlyzko/unpublished/index.html.

[59] K. Ramachandra, Some remarks on the mean value of the Riemann zeta-function and other Dirichlet series - i, Hardy-Ramanujan J., 1:1-15, 1978.

[60] K. Ramachandra, Some remarks on the mean value of the Riemann zeta-function and other Dirichlet series - ii, Hardy-Ramanujan J., 3:1-24, 1980.

[61] K. Ramachandra, Some remarks on the mean value of the Riemann zeta-function and other Dirichlet series - iii, Ann. Acad. Sci. Fenn. Ser. AI Math., 5:145-158, 1980.

[62] Z. Rudnick and P. Sarnak, Zeros of principal $L$-functions and random matrix theory, Duke Math. J., 81(2):269-322, 1996.

[63] K. Soundararajan, Mean values of the Riemann zeta-function, Mathematika, 42:158174, 1995.

[64] K. Soundararajan, Extreme values of zeta and L-functions, Math. Ann., 342:467-86, 2008.

[65] K. Soundararajan, Moments of the Riemann zeta-function, Ann. of Math., 170(2):98193, 2009, arXiv:math/0612106. 
[66] J. Stopple, The quadratic character experiment, Experiment. Math., 18(2):193-200, 2009, arXiv:0802.4255.

[67] E.C. Titchmarsh, The Theory of the Riemann Zeta Function, Clarendon Press, Oxford, second edition, 1986.

[68] H. Weyl, Classical Groups, Princeton University Press, 1946. 